\title{
A cut finite element method for the solution of the full-potential equation with an embedded wake
}

\author{
M.Davari ${ }^{1}$ • R.Rossi ${ }^{1}, 2$ • P. Dadvand ${ }^{2}$ • I. López ${ }^{3}$ • R. Wüchner ${ }^{3}$
}

Received: date / Accepted: date

\begin{abstract}
Potential flow solvers represent an appealing alternative for the simulation of non-viscous subsonic flows. In order to deliver accurate results, such techniques require prescribing explicitly the so called Kutta condition, as well as adding a special treatment on the "wake" of the body. The wake is traditionally modelled by introducing a gap in the CFD mesh, which requires an often laborious meshing work. The novelty of the proposed work is to embed the wake within the CFD domain. The approach has obvious advantages in the context of aeroelastic optimization, where the position of the wake may change due to evolutionary steps of the geometry. This work presents a simple, yet effective, method for the imposition of the embedded wake boundary condition. The presented method preserves the possibility of employing iterative techniques in the solution of the linear problems which stem out of the discretization. Validation and verification of the solver are performed for a NACA 0012 airfoil.
\end{abstract}

Keywords Full potential solver · Extended finite element method $\cdot$ Kutta condition

\section{Introduction}

Fluid solvers are a key component in aircraft optimization workflows. When choosing the right solver for a certain application, properties such as accuracy, computational effort and robustness have to be considered.

M.Davari

E-mail: md_civil1983@yahoo.com

${ }^{1}$ Polytechnic University of Catalonia (UPC), Barcelona, Spain. .

${ }^{2}$ International Centre for Numerical Methods in Engineering,

Barcelona, Spain .

${ }^{3}$ Technical University of Munich, Munich, Germany
Currently, the tendency is to integrate the optimization workflows in early stages of aircraft design, where fast solutions are required. Despite the large growth of computer power, there is still a significant overhead due to the large amount of design variables and system evaluations required to achieve accurate results. Most parts of the overhead can be attributed to the fluid solvers. For this reason, every effort is made to develop low-fidelity fluid solvers to reduce the computational cost.

This paper presents an innovative finite element fullpotential solver with an embedded wake approach. The advantage of full-potential solvers is that only a single, scalar, partial-differential equation (conservation of mass) is needed to obtain flow solutions, instead of five coupled equations (conservation of mass, momentum, energy). Therefore, for a given mesh the number of variables is five times lower than for the solution of the Euler equations [1]. Potential solvers can reproduce flow solutions with shock waves, such as in transonic $[2,3,4,5,6]$ and supersonic $[7,8,9,10]$ flows. However, in the current study only subsonic flows are considered $(M<1)$. Thus, the fluid stays subsonic everywhere. In this case there are no shock waves, but the jump across the wake still needs to be modeled.

The full-potential equation can be derived from the Navier Stokes equations assuming steady, irrotational and isentropic flow. These assumptions hold for the flow outside the boundary layer of streamlined bodies flying at high Reynolds numbers and small angles of attack (i.e. with attached boundary layers). For benign subsonic flows, the full-potential equation is elliptic but for some localized regions of high flow curvature. Thus, the finite element method provides robust and accurate results for this range of application.

The full-potential formulation requires a model to approximate the wake shed behind the body, where the 
flow field is not irrotational. In reality, the wake exhibits a roll up, which can be computed if the unsteady terms are kept in the formulation. Otherwise, a model for the geometry of the wake has to be introduced. Typically the wake is assumed to be straight and follow the direction of the freestream velocity. This assumption is relaxed imposing a continuous mass flux across the wake. Furthermore, the wake cannot support pressure jumps. This is ensured by means of the pressure-equality wakeboundary condition.

Moreover, for very high Reynold numbers the wake can be approximated as a discontinuity. Hence, across the wake the fluid magnitudes and their derivatives may present finite jumps. Particularly, the potential exhibits a jump across the wake. In order to support the jump in the potential, Nishida and Drela [11] developed a finite element method in which elements are not connected across the wake surface. David Eller improved the method's flexibility and robustness by enforcing the pressure-equality wake-boundary condition using a least-squares approach [1, 12]. However, both methods require a body-fitted mesh around the wake. This is a major disadvantage when performing aeroe-

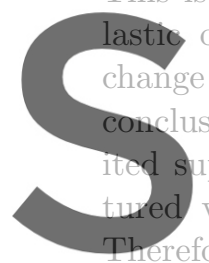
optimization, where the
during the evolutionary
sions of Eller's work it is st
pport for the automatic g
volume meshes with ernb
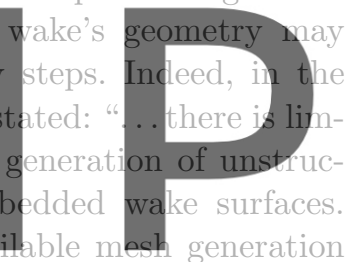

software to robustly create element meshes including

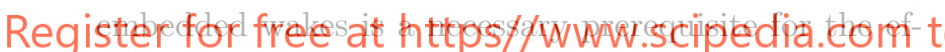
fective use of the method..." [1]. Based on this, the present paper is focused on the development of an embedded wake approach.

The eXtended finite element method (X-FEM) is widely used to embed discontinuities $[13,14,15,16,17$, 18, 19, 20]. In X-FEM special functions are added to the finite element approximation space in order to be able to capture discontinuities within the element. One of the main disadvantages of standard X-FEM is that these additional special functions should be considered not only for the elements that contain the discontinuity, but also for those elements adjacent to them (blending elements). There are various research studies aiming to avoid blending elements [21, 22, 23, 24, 25, 26, 27]. In [21, 22] a method to capture strong and weak discontinuities by means of an explicit variable is presented. In [23] the method is improved by adding new functions to the cut elements. The main achievement of [23] is that the additional functions are controlled by new local degrees of freedom, which can be condensed before final assembly without changing the graph and the size of the final system. A further method that allows embed- ding discontinuities is the mesh-independent interfaceenriched generalized finite element method (IGFEM) [24]. In IGFEM the element is enriched by adding nodes on the discontinuity. [25] improved IGFEM in order to reduce the discretization error on curved interfaces. IGFEM can handle weak discontinuities associated with multiple material interfaces crossing a single element [26]. Adding an extra enrichment function to the solution space allows to also handle strong discontinuities. This last approach is denoted discontinuity-enriched finite element method (D-EFEM) [27].

In the presented method the element nodes are duplicated. The auxiliary nodes allow to capture the wake discontinuity by decoupling the degrees of freedom above the wake from the degrees of freedom below the wake.

At the same time, the auxiliary nodes allow to apply the wake-boundary conditions. The advantages of this method are:

\section{Blending elements are not necessary. An explicit variable is not required. The graph of the final system does not change. The system remains well conditioned, hence retain-}

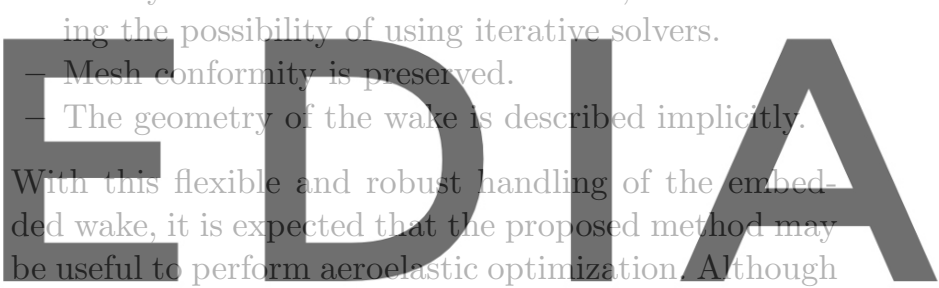

this paper is concerned with the embedded modelling of the mel ad the version withoute the watermark technique with the methods described in 28), in or der to use a fully immersed description of the geometry of interest. In this work the wake boundary conditions are applied using a least-squares finite-element approach. The solver is implemented in Kratos MultiPhysics, an open source framework to develop multidisciplinary programs [29]. Efficiency is achieved using Kratos' object-oriented data structure. Validation and verification of the solver are performed for a NACA 0012.

\section{Method}

\subsection{Governing Equation}

The flow outside the boundary layer of streamlined bodies flying at high Reynold numbers and small angles of attack, can be assumed to be steady, irrotational and isentropic [30]. This allows to reduce the Navier-Stokes equations to the steady full-potential equation

$\nabla \cdot(\rho \nabla \Phi)=0$ 
where $\rho$ is the density and $\Phi$ is the velocity potential. Eq.(1) is a nonlinear partial differential equation for the potential, which expresses the conservation of mass. The compressible Bernoulli equation states the nonlinear relation between the density and the potential:

$\rho=\rho_{\infty}\left[1+\frac{\gamma-1}{2} \frac{u_{\infty}^{2}}{a_{\infty}^{2}}\left(1-\frac{\nabla \Phi \cdot \nabla \Phi}{u_{\infty}^{2}}\right)\right]^{\frac{1}{\gamma-1}}$

where $u_{\infty}, \rho_{\infty}$ and $a_{\infty}$ are the freestream velocity, density and speed of sound respectively and $\gamma$ is the ratio of specific heats. The pressure $p$ can be expressed in terms of the density and the far-field quantities using the following isentropic relation:
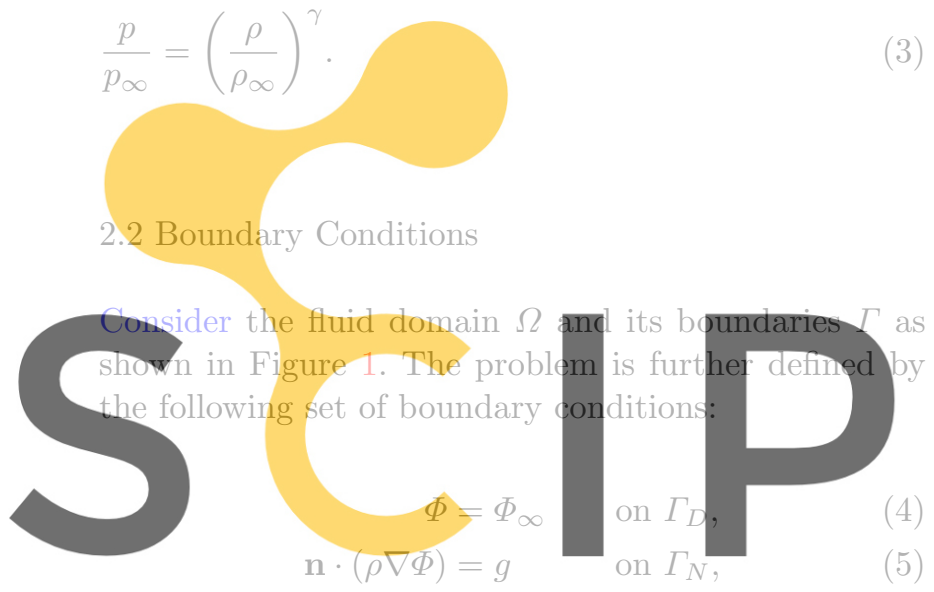

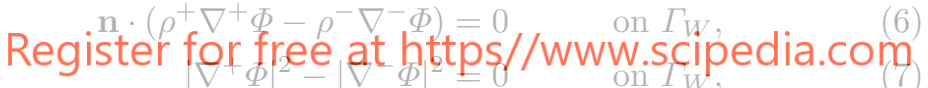

where $\mathbf{n}$ is the surfaces' normal and the subscripts + and - denote the upper and lower wake respectively. Eq.(4) is a Dirichlet condition, which specifies the value of the far-field potential $\Phi_{\infty}$ at the inlet. Note that this condition is required to determine the absolute value of the potential because the full-potential equation and the remaining boundary conditions are defined in terms of its derivatives only. Eq.(5) is a Neumann condition, which imposes the mass flux on $\Gamma_{N}$. On the wing and on the walls a slip boundary condition is enforced because viscosity is neglected $(g=0)$. On the outlet, the free stream velocity is specified as a Neumann condition with $g=\mathbf{n} \cdot\left(\rho_{\infty} \nabla \Phi_{\infty}\right)$. A Dirichlet condition cannot be applied on the outlet because there is a jump in the potential across the wake and the wake intersects the outlet (Figure 1). Eqs.(6) and (7) are the wake boundary conditions. Eq.(6) imposes mass conservation across the wake, relaxing the straight wake assumption and Eq.(7) imposes equality of pressures between the upper and lower wake.

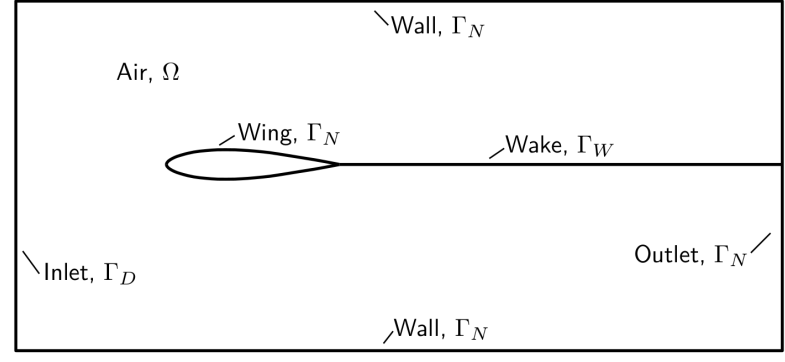

Fig. 1: Fluid domain $\Omega$ and its boundary $\Gamma$.

\subsection{Discretization}

The weak form of the system is obtained applying the Galerkin weighted residual method and the divergence theorem to Eq.(1):

$\int_{\Omega} \rho \nabla \omega \cdot \nabla \Phi d \Omega=\int_{\Gamma_{N}} \omega \mathbf{n} \cdot(\rho \nabla \Phi) d \Gamma$,

where $\omega$ is the test function. To define the weak finiteelement form from Eq.(8), the domain of interest $\Omega$ is divided into simplicial finite elements. The method is

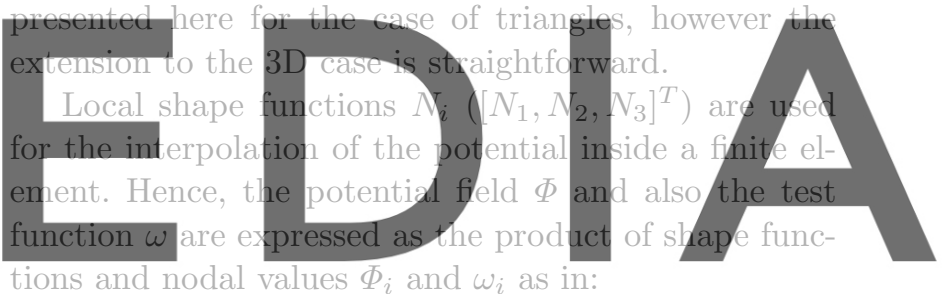

tions and nodal values $\Phi_{i}$ and $\omega_{i}$ as in:

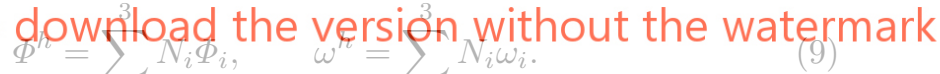 \\ $i=1$}

Therefore, the weak finite-element form of the problem can be obtained by substituting Eq.(9) into Eq.(8):

$\sum_{i=1}^{3} \sum_{j=1}^{3} \int_{\Omega} \rho \nabla N_{i} \cdot \nabla N_{j} d \Omega \Phi_{j}=\sum_{i=1}^{3} \int_{\Gamma_{N}} N_{i} g d \Gamma$,

where the right-hand side has been replaced by the Neumann boundary condition (Eq.(5)). Eq.(10) can be expressed in matrix form for a finite triangular element as:

$\mathbf{K}_{e}^{(3 \times 3)} \boldsymbol{\Phi}_{e}^{(3 \times 1)}=\mathbf{F}_{e}^{(3 \times 1)}$.

Since, the density is a nonlinear function of the potential, the discrete form of the problem is nonlinear. Hence, Eq.(10) is rewritten in residual form $\mathbf{R}(\Phi)=0$, where the contribution of each element $e$ to the residual is:

$$
R_{i}=\sum_{j=1}^{3} \int_{\Omega_{e}} \rho \nabla N_{i} \cdot \nabla N_{j} d \Omega \Phi_{j}-\int_{\Gamma_{N e}} N_{i} g d \Gamma=0 .
$$


The global nonlinear problem is then solved by means of the Newton's method. In this way the solution is updated by an increment $\Delta \Phi^{k}$, which can be computed from:

$\mathbf{J} \Delta \boldsymbol{\Phi}^{k}=-\mathbf{R}\left(\boldsymbol{\Phi}^{k}\right)$,

where the entries $J_{i, j}$ of the Jacobian matrix $\mathbf{J}$ are defined by

$$
\begin{aligned}
J_{i, j}=\frac{\partial R_{i}(\mathbf{\Phi})}{\partial \Phi_{j}} & =\int_{\Omega_{e}} \rho \nabla N_{i} \cdot \nabla N_{j} \\
& +2 \frac{\partial \rho}{\partial|\mathrm{u}|^{2}}\left(\nabla N_{j} \cdot \nabla \Phi\right)\left(\nabla N_{i} \cdot \nabla \Phi\right) d \Omega
\end{aligned}
$$

and the derivative of the density with respect to the local velocity is:

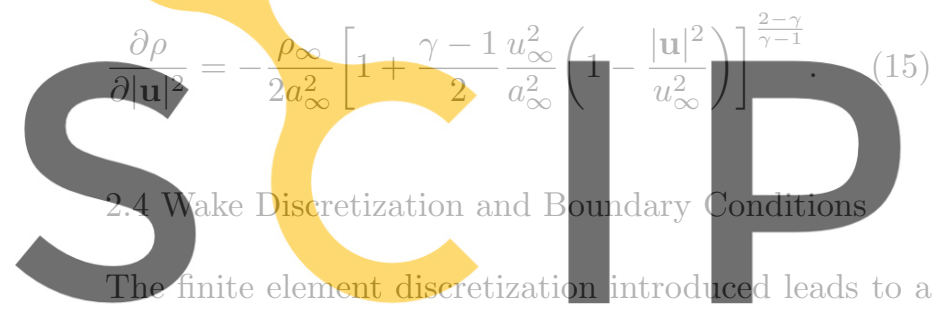

non-lifting problem $[31,32]$. In order to enable the gen-

Register for freulation areund the body the walke boundary conditions (Eqs. (6) and (7) must be enforced. Ihe embedded wake method are presented in this section (Figure 2).

\subsubsection{Standard body-fitted wake method}

Let us begin by describing the standard approach, in which the wake is modelled as an infinitely thin gap. This gap introduces a geometrical boundary in the CFD domain. Following the method presented in [1], to support a jump in the value of the potential across the wake, finite elements are not connected across the wake surface (Figure 2). Elements that share a node with the wake are only attached to one side of the wake, so that all wake nodes must be present at least twice. Without any further boundary conditions, the wake acts as a solid wall. The mass-flux boundary condition is enforced using a Galerkin finite element method. The weak form of Eq.(6) is:

$\int_{\Gamma_{w}} \omega\left(\rho^{+} \nabla \Phi^{+} \cdot \mathbf{n}^{+}+\rho^{-} \nabla \Phi^{-} \cdot \mathbf{n}^{-}\right) d \Gamma=0$, where $\mathbf{n}^{+}$and $\mathbf{n}^{-}$are upper and lower wake normal vectors respectively. Using Eq.(10) the wake surface integral can be expressed in terms of a volume integral:

$$
\int_{\Omega} \rho^{+} \nabla \omega^{+} \cdot \nabla \Phi^{+}+\rho^{-} \nabla \omega^{-} \cdot \nabla \Phi^{-} d \Omega=0 .
$$

Discretizing into finite elements yields:

$R_{i}^{w}=\sum_{j=1}^{n_{w}} \int_{\Omega_{j}} \rho \nabla N_{i} \cdot \nabla \Phi d \Omega=0$.

where the summation is performed over the elements that share a wake node. The entries $J_{i, j}^{w}$ of the Jacobian matrix are defined for each element:

$$
\begin{aligned}
J_{i, j}^{w}=\frac{\partial R_{i}^{w}(\Phi)}{\partial \Phi_{j}} & =\int_{\Omega_{e}} \rho \nabla N_{i} \cdot \nabla N_{j} \\
& +2 \frac{\partial \rho}{\partial|\mathbf{u}|^{2}}\left(\nabla N_{j} \cdot \nabla \Phi\right)\left(\nabla N_{i} \cdot \nabla \Phi\right) d \Omega .
\end{aligned}
$$

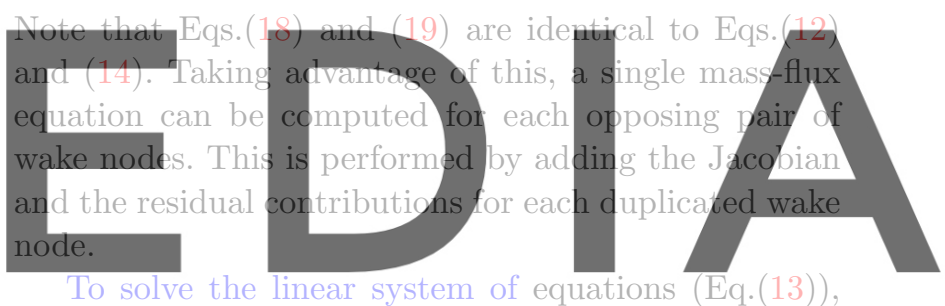

the number of equations. must be equal to the num-

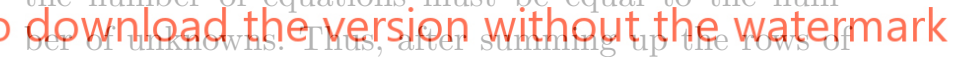

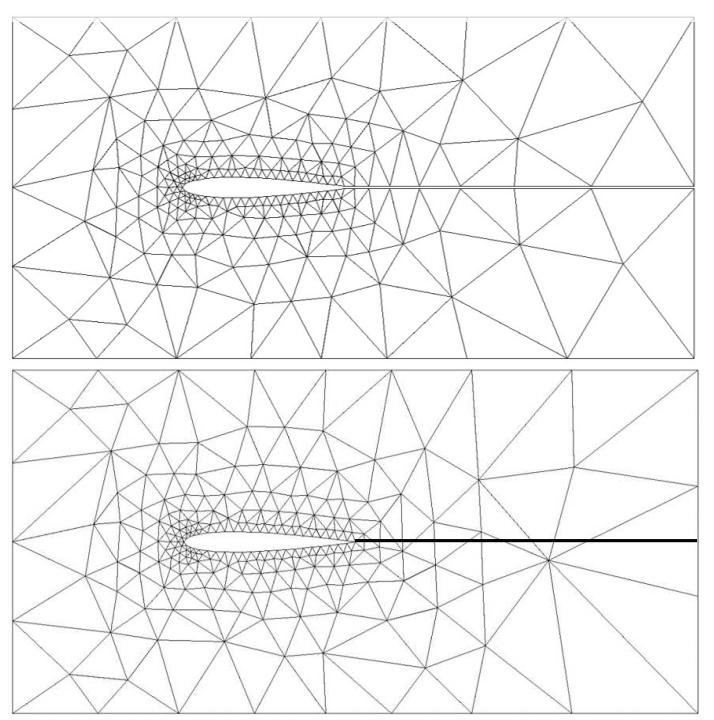

Fig. 2: Sketch of a standard body fitted approach on the up and the proposed wake embedded approach on the down. 
each pair of wake nodes, the upper nodes equations are canceled and they are replaced with pressure-equality wake-boundary condition (Eq.(7)), which can be rewritten as:

$\mathbf{u}^{+} \cdot \nabla \Phi^{+}-\mathbf{u}^{-} \cdot \nabla \Phi^{-}=0$.

Assuming small-disturbance flows, the total velocities $\mathbf{u}^{+}$and $\mathbf{u}^{-}$can be expressed as follows:

$\mathbf{u}^{+}=\mathbf{u}_{\infty}(1+u)$

$\mathbf{u}^{-}=\mathbf{u}_{\infty}(1+u)$,

where $u$ denotes the perturbation velocity and is assumed to be $u \ll 1$. Making use of Eqs.(21) and (22) and dropping high order terms allows to linearize the pressure-equality wake-boundary condition:

$\mathbf{u}_{\infty} \cdot \nabla\left(\Phi^{+}-\Phi^{-}\right)=0$

which is a convection equation for the potential jump across the wake. Eq.(23) states that the potential jump is constant along the wake. Using a least-squares approach leads to the residual energy functional

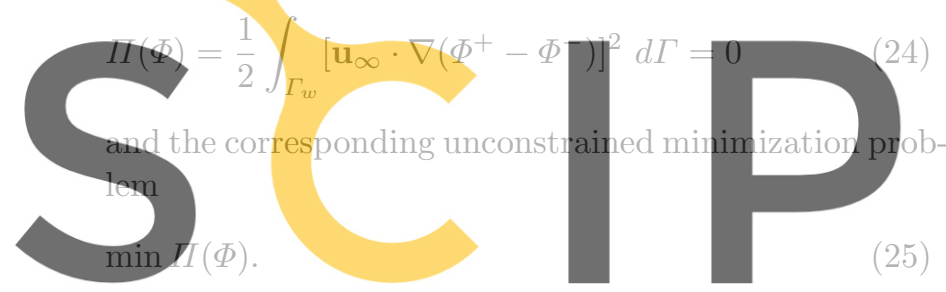

Introducing the finite element discretization (Eq.(9))

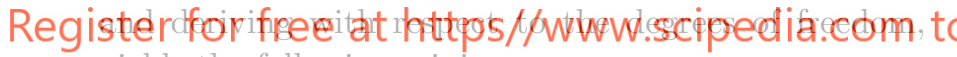
yields the following minimum:

$$
\begin{aligned}
& R_{i}^{p}=\frac{\partial \Pi(\overline{\mathbf{\Phi}})}{\partial \Phi_{i}} \\
& =\sum_{e=1} \int_{\Gamma_{w, e}}\left[\mathbf{u}_{\infty} \cdot \nabla N_{i}\right]\left[\mathbf{u}_{\infty} \cdot\left(\nabla \mathbf{N}^{+} \boldsymbol{\Phi}^{+}-\nabla \mathbf{N}^{-} \boldsymbol{\Phi}^{-}\right)\right] d \Gamma \\
& =0
\end{aligned}
$$

Second differentiation with respect to the degrees of freedom results in the corresponding Jacobian:

$$
\begin{aligned}
J_{i, j}^{p} & =\frac{\partial R_{i}^{p}}{\partial \Phi_{j}} \\
& =\sum_{e=1} \int_{\Gamma_{w, e}}\left[\mathbf{u}_{\infty} \cdot \nabla N_{i}\right]\left[\mathbf{u}_{\infty} \cdot\left(\nabla \mathbf{N}_{j, u}-\nabla \mathbf{N}_{j, l}\right)\right] d \Gamma=0
\end{aligned}
$$

which completes the equation $J_{i, j}^{p} \Phi_{j}=-R_{i}^{p}$ for each of the wake nodes. Note that before modifying the global stiffness matrix in order to enforce the wake boundary conditions, the matrix was symmetric and positive definite. After summing up the rows of each pair of wake nodes (Eqs.(18) and (19)) and replacing the upper nodes equations with the pressure-equality wakeboundary condition (Eqs.(26) and (27)), the matrix is no longer symmetric. Moreover, this standard body fitted wake treatment requires additional effort in the mesh-generation process in order to deal with the gap introduced in the domain. While this might not represent a major issue at the preprocessing stage, it becomes a potential blocker if the shape of the wake can evolve in time, as may happen for example due to control operations or within an optimization loop. In this context, a change of the geometry of the wake cannot be easily handled, particularly in the case in which the wake intersects other bodies (for example the fuselage). This motivates the use of the proposed embedded wake treatment presented in the next section.

\subsubsection{Proposed embedded wake method}

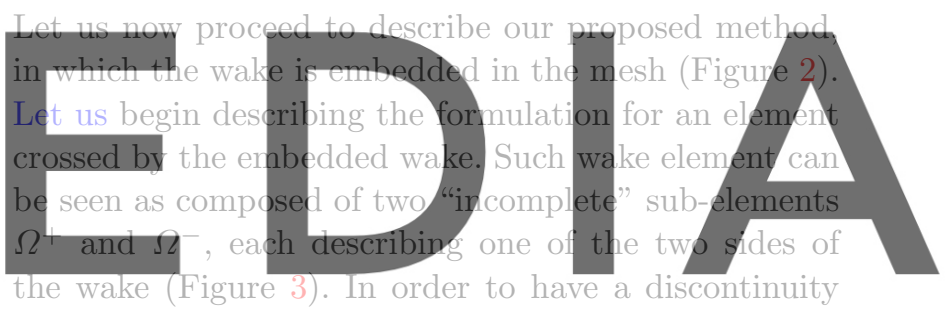

in the potential field across the wake, the potential is

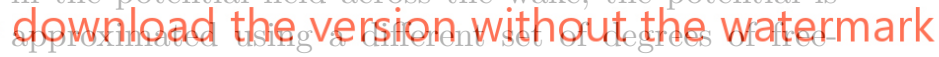

dom in each sub-element. Three new auxiliary degrees of freedom $\Psi_{i}$, located at the position of the nodes of the mesh are therefore introduced. For further insight, assume that $\Theta^{+}$and $\Theta^{-}$identify the sets of degrees of freedom corresponding to elements $\Omega^{+}$and $\Omega^{-}$respectively. Then the degrees of freedom, corresponding with each sub-element are:

$\Theta_{i}^{+}=\left\{\begin{array}{l}\Phi_{i} \text { if } i^{t h} \text { node } \in \Omega^{+} \\ \Psi_{i} \text { if } i^{t h} \text { node } \in \Omega^{-}\end{array}\right.$,

$\Theta_{i}^{-}=\left\{\begin{array}{l}\Psi_{i} \text { if } i^{t h} \text { node } \in \Omega^{+} \\ \Phi_{i} \text { if } i^{t h} \text { node } \in \Omega^{-}\end{array}\right.$.

Figure 3 shows the set of degrees of freedom of a wake element. Inside the wake element, the standard finite element shape functions $\mathbf{N}$ are used twice. Thus, the wake element is effectively duplicated. The new expanded wake element shape functions $\widetilde{\mathbf{N}}$ are defined on the ba- 

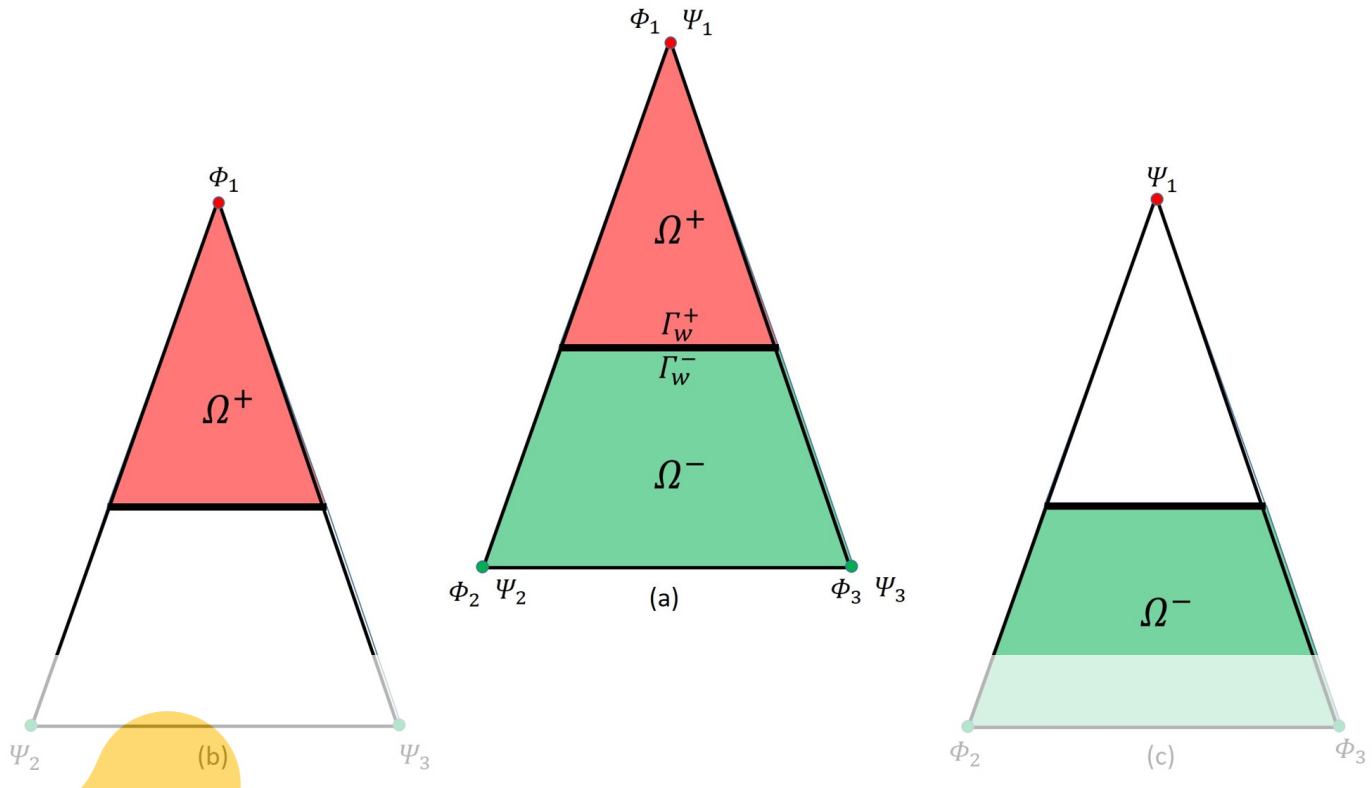

Fig. 3: Degrees of freedom of a cut element. (a) shows a cut element with all its degrees of freedom. (b) and (c) show the degrees of freedom corresponding to $\Omega^{+}$and $\Omega^{-}$respectively.

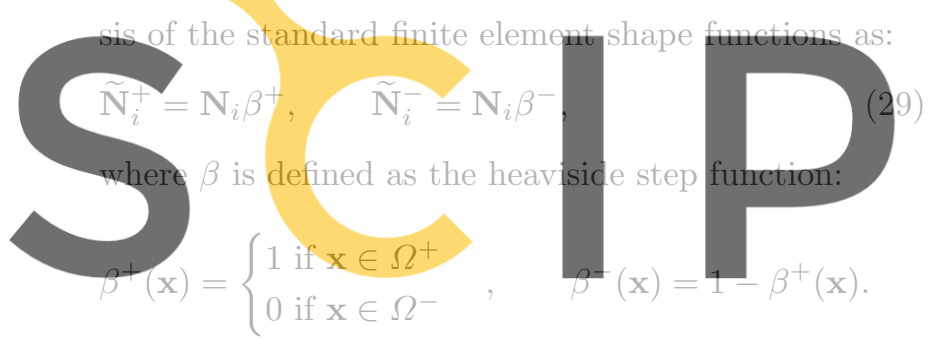

Register for free at https//www.scipedia.copp) to

Note that the new expanded shape functions are defined in such a manner that no relation exists between the degrees of freedom $\Theta^{+}$and $\Theta^{-}$. This yields a new approximation for the potential and the test function:

$$
\begin{aligned}
\Theta^{+} & =\sum_{i=1}^{3} \widetilde{N}_{i}^{+} \Theta_{i}^{+}, & \Theta^{-} & =\sum_{i=1}^{3} \widetilde{N}_{i}^{-} \Theta_{i}^{-}, \\
\omega^{+} & =\sum_{i=1}^{3} \widetilde{N}_{i}^{+} \omega_{i}^{+}, & \omega^{-} & =\sum_{i=1}^{3} \widetilde{N}_{i}^{-} \omega_{i}^{+} .
\end{aligned}
$$

Applying this discretization and assuming for simplicity triangular elements, Eq.(10) can be rewritten for the upper and lower sub-elements as:

$\sum_{i=1}^{3} \sum_{j=1}^{3} \int_{\Omega_{w}^{+}} \rho^{+} \nabla \tilde{\mathbf{N}}_{i}^{+} \cdot \nabla \tilde{\mathbf{N}}_{j}^{+} d \Omega \Theta_{j}^{+}=\sum_{i=1}^{3} \int_{\Gamma_{w}^{+}} \omega^{+} g^{+} d \Gamma$,

$\sum_{i=1}^{3} \sum_{j=1}^{3} \int_{\Omega_{w}^{-}} \rho^{-} \nabla \tilde{\mathbf{N}}_{i}^{-} \cdot \nabla \tilde{\mathbf{N}}_{j}^{-} d \Omega \Theta_{j}^{-}=\sum_{i=1}^{3} \int_{\Gamma_{w}^{-}} \omega^{-} g^{-} d \Gamma$.

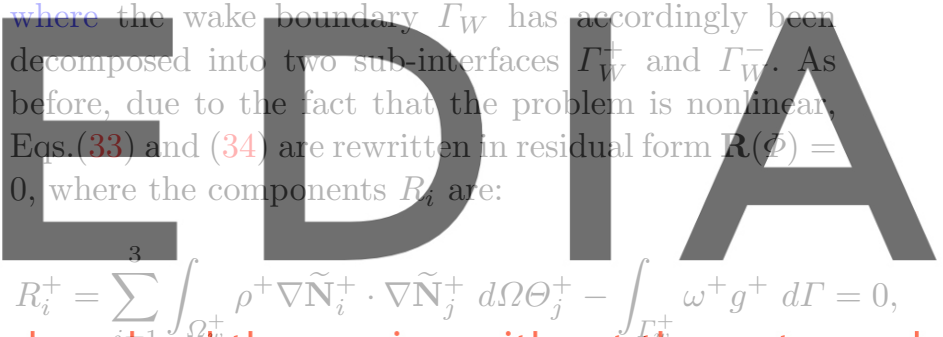

$R_{i}^{+}=\sum_{i} \int_{\Omega^{+}} \rho^{+} \nabla \widetilde{\mathbf{N}}_{i}^{+} \cdot \nabla \widetilde{\mathbf{N}}_{j}^{+} d \Omega \Theta_{j}^{+}-\int_{j} \omega^{+} g^{+} d \Gamma=0$,
dowifoad the version without the watermark

$$
R_{i}^{-}=\sum_{j=1}^{3} \int_{\Omega_{w}^{-}} \rho^{-} \nabla \tilde{\mathbf{N}}_{i}^{-} \cdot \nabla \tilde{\mathbf{N}}_{j}^{-} d \Omega \Theta_{j}^{-}-\int_{\Gamma_{w}^{-}} \omega^{-} g^{-} d \Gamma=0
$$

Note that the residual terms of Eqs.(35) and (36) are identical to Eq.(12). Thus, the Jacobian terms stemming out of Eqs.(35) and (36) can be obtained from Eq.(14). The resulting linear system of equations can be written in matrix form as:

$$
\left[\begin{array}{cc}
\mathbf{J}^{+(3 \times 3)} & 0 \\
0 & \mathbf{J}^{-(3 \times 3)}
\end{array}\right]\left[\begin{array}{c}
\Delta \boldsymbol{\Theta}^{+(3 \times 1)} \\
\Delta \boldsymbol{\Theta}^{-(3 \times 1)}
\end{array}\right]=-\left[\begin{array}{l}
\mathbf{R}^{+(3 \times 1)} \\
\mathbf{R}^{-(3 \times 1)}
\end{array}\right] .
$$

For the element in Figure 3, Eq.(37) can be expressed as:

$$
\left[\begin{array}{cccccc}
J_{11}^{+} & J_{12}^{+} & J_{13}^{+} & 0 & 0 & 0 \\
J_{21}^{+} & J_{22}^{+} & J_{23}^{+} & 0 & 0 & 0 \\
J_{11}^{+} & J_{11}^{+} & J_{11}^{+} & 0 & 0 & 0 \\
0 & 0 & 0 & J_{11}^{-} & J_{12}^{-} & J_{13}^{-} \\
0 & 0 & 0 & J_{21}^{-} & J_{22}^{-} & J_{23}^{-} \\
0 & 0 & 0 & J_{11}^{-} & J_{11}^{-} & J_{11}^{-}
\end{array}\right]\left[\begin{array}{l}
\Phi_{1} \\
\Psi_{2} \\
\Psi_{3} \\
\Psi_{4} \\
\Phi_{5} \\
\Phi_{6}
\end{array}\right]=-\left[\begin{array}{c}
R_{1}^{+} \\
R_{2}^{+} \\
R_{3}^{+} \\
R_{1}^{-} \\
R_{2}^{-} \\
R_{3}^{-}
\end{array}\right]
$$


Observe that the degrees of freedom of upper and lower wake sub-elements are fully decoupled in Eq.(38). An additional condition needs to be applied to impose the relation between $\Theta^{+}$and $\Theta^{-}$.

Imposition of the Wake Boundary Conditions Let us consider a two dimensional problem where the wake boundary conditions Eqs.(6) and (7) can be rewritten as a single vector equation:

$\rho^{+} \nabla \Theta^{+}-\rho^{-} \nabla \Theta^{-}=0$.

Applying a least squares finite element approach yields the residual energy functional

$\Pi(\Theta)=\frac{1}{2} \int_{\Gamma_{\omega}}\left[\rho^{+} \nabla \Theta^{+}-\rho^{-} \nabla \Theta^{-}\right]^{2} d \Gamma=0$,

where $\Gamma_{w}=\Gamma_{w}^{+} \oplus \Gamma_{w}^{-}$, and the corresponding unconstrained minimization problem

$\min \Pi(\Theta)$.

Discretizing into finite elements and deriving with respect to the degrees of freedom yields the minimum:

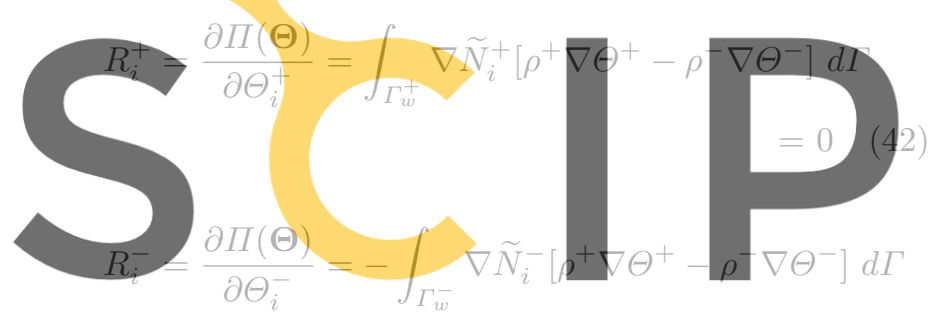

Register for free at https//www.scipedia.com where the small-disturbance assumption $u \ll 1$ allows to neglect the derivatives of the density. This is a good approximation on the wake, where compressible effects are small. Moreover, note that the following integrals vanish since:

$\int_{\Gamma_{w}^{+}} \rho^{+} \nabla \tilde{N}_{i}^{+} \cdot \nabla \tilde{N}_{j}^{-} d \Gamma=\int_{\Gamma_{w}^{-}} \rho^{-} \nabla \tilde{N}_{i}^{-} \cdot \nabla \widetilde{N}_{j}^{+} d \Gamma=0$,

which can be derived from Eqs.(29) and (30). Making use of Eq.(44) and deriving Eqs.(42) and (43) with respect to the degrees of freedom yields the contributions for upper and lower sub-elements' Jacobians respectively:

$$
\begin{aligned}
J_{i}^{+}=\frac{\partial R_{i}^{+}}{\partial \Theta_{j}^{+}} & =\int_{\Gamma_{w}^{+}} \rho^{+} \nabla \tilde{N}_{i}^{+} \cdot \nabla \tilde{N}_{j}^{+} \\
& +2 \frac{\partial \rho^{+}}{\partial|\mathbf{u}|^{2}}\left(\nabla \tilde{N}_{j}^{+} \cdot \nabla \Theta^{+}\right)\left(\nabla \tilde{N}_{i}^{+} \cdot \nabla \Theta^{+}\right) d \Gamma=0
\end{aligned}
$$

$$
\begin{aligned}
J_{i}^{-}=\frac{\partial R_{i}^{-}}{\partial \Theta_{j}^{-}}= & -\int_{\Gamma_{w}^{-}} \rho^{-} \nabla \tilde{N}_{i}^{-} \cdot \nabla \widetilde{N}_{j}^{-} \\
& +2 \frac{\partial \rho^{-}}{\partial|\mathbf{u}|^{2}}\left(\nabla \widetilde{N}_{j}^{-} \cdot \nabla \Theta^{-}\right)\left(\nabla \widetilde{N}_{i}^{-} \cdot \nabla \Theta^{-}\right) d \Gamma=0 .
\end{aligned}
$$

Due to the fact that the shape functions are linear within the sub-elements, their gradient is constant. Therefore, the surface integral introduced in Eqs.(45) and (46) can be expresed in terms of a volume integral as:

$$
\begin{aligned}
J_{i}^{+}=\frac{\partial R_{i}^{+}}{\partial \Theta_{j}^{+}}= & \int_{\Omega_{w}^{+}} \rho^{+} \nabla \widetilde{N}_{i}^{+} \cdot \nabla \widetilde{N}_{j}^{+} \\
& +2 \frac{\partial \rho^{+}}{\partial|\mathrm{u}|^{2}}\left(\nabla \widetilde{N}_{j}^{+} \cdot \nabla \Theta^{+}\right)\left(\nabla \widetilde{N}_{i}^{+} \cdot \nabla \Theta^{+}\right) d \Omega=0,
\end{aligned}
$$

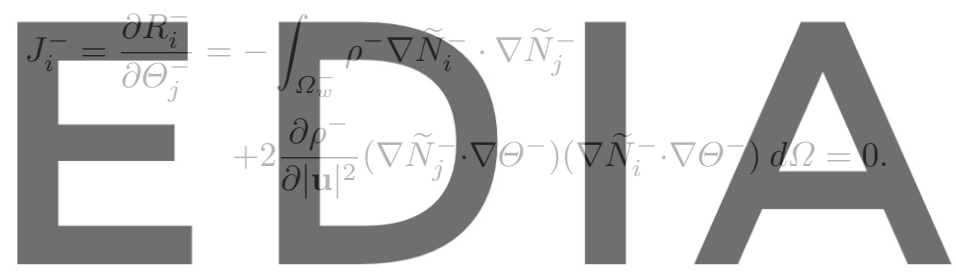

(48)

o download the version without the watermark boundary condition are identical to the Jacobian terms obtained from the funl-potential equation (Eq.(14)). As mentioned earlier $\Omega_{w}=\Omega_{w}^{+} \oplus \Omega_{w}^{-}$. This allows to rewrite Eqs.(47) and (48) as:

$\frac{\partial R_{i}^{+}}{\partial \Theta_{j}^{+}} \Delta \Theta_{j}^{+}+\frac{\partial R_{i}^{-}}{\partial \Theta_{j}^{-}} \Delta \Theta_{j}^{-}=-R_{i}\left(\Theta^{+}, \Theta^{-}\right)$,

which can be expressed in matrix form for each element as:

$$
\left[\mathbf{B}^{+(3 \times 3)} \mathbf{B}^{-(3 \times 3)}\right]\left[\begin{array}{c}
\Delta \boldsymbol{\Theta}^{+(3 \times 1)} \\
\Delta \boldsymbol{\Theta}^{-(3 \times 1)}
\end{array}\right]=-\left[\mathbf{R}^{(3 \times 1)}\right] .
$$

Consider again the element presented in Figure 3. For this element Eq.(50) can be rewritten as:

$\left[\begin{array}{llllll}B_{11}^{+} & B_{12}^{+} & B_{13}^{+} & B_{11}^{-} & B_{12}^{-} & B_{13}^{-} \\ B_{21}^{+} & B_{22}^{+} & B_{23}^{+} & B_{21}^{-} & B_{22}^{-} & B_{23}^{-} \\ B_{31}^{+} & B_{32}^{+} & B_{33}^{+} & B_{31}^{-} & B_{32}^{-} & B_{33}^{-}\end{array}\right]\left[\begin{array}{l}\Phi_{1} \\ \Psi_{2} \\ \Psi_{3} \\ \Psi_{4} \\ \Phi_{5} \\ \Phi_{6}\end{array}\right]=-\left[\begin{array}{c}R_{1}^{-} \\ R_{2}^{+} \\ R_{3}^{+}\end{array}\right]$. 
This represents the constraint to be respected. The wake boundary conditions are applied substituting Eq.(51) a by the equations corresponding to the auxiliary degrees of freedom $\Psi_{i}$ in Eq.(38):

$$
\left[\begin{array}{cccccc}
J_{11}^{+} & J_{12}^{+} & J_{13}^{+} & 0 & 0 & 0 \\
B_{21}^{+} & B_{22}^{+} & B_{23}^{+} & B_{21}^{-} & B_{22}^{-} & B_{23}^{-} \\
B_{31}^{+} & B_{32}^{+} & B_{33}^{+} & B_{31}^{-} & B_{32}^{-} & B_{33}^{-} \\
B_{11}^{+} & B_{12}^{+} & B_{13}^{+} & B_{11}^{-} & B_{12}^{-} & B_{13}^{-} \\
0 & 0 & 0 & J_{21}^{-} & J_{22}^{-} & J_{23}^{-} \\
0 & 0 & 0 & J_{11}^{-} & J_{11}^{-} & J_{11}^{-}
\end{array}\right]\left[\begin{array}{l}
\Phi_{1} \\
\Psi_{2} \\
\Psi_{3} \\
\Psi_{4} \\
\Phi_{5} \\
\Phi_{6}
\end{array}\right]=-\left[\begin{array}{c}
R_{1}^{+} \\
R_{2}^{+} \\
R_{3}^{+} \\
R_{1}^{-} \\
R_{2}^{-} \\
R_{3}^{-}
\end{array}\right]
$$

\section{Computational Results}

To validate and verify the implemented embedded wake method, the results of the flow over a symmetric NACA 0012 airfoil are presented. First, a mesh-convergence study comparing the embedded wake method with the body fitted method is performed. Afterwards, the numerical results are compared with experimental data.
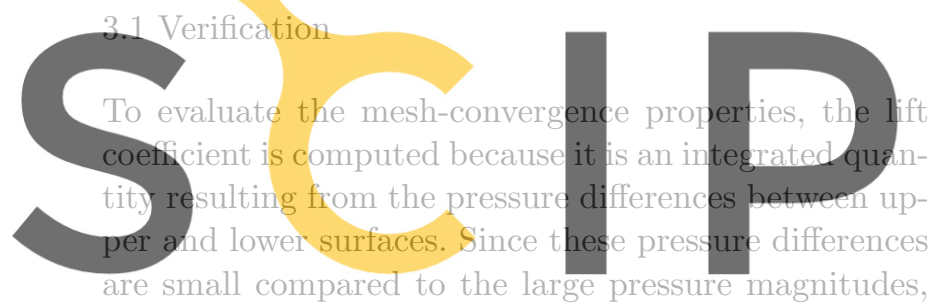

the lift coefficient is very sensitive to numericol orros

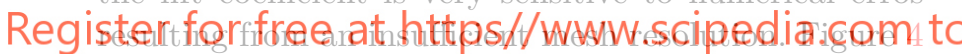
shows the value of the lift coefficient over the number of degrees of freedom for an angle of attack $\alpha=15^{\circ}$ and a free-stream Mach number $M_{\infty}=0.15$. Note that for $2.3 \cdot 10^{4}$ degrees of freedom the lift coefficient is already converged. The results for both methods display large differences for a low number of degrees of freedom.

\subsection{Validation}

To validate the embedded wake method, the pressure coefficient distribution is compared against experimental data in order to establish the ability of the embedded wake method to reproduce physics. The results for two angles of attack $\alpha=0^{\circ}$ and $\alpha=15^{\circ}$ and a free-stream Mach number $M_{\infty}=0.15$ are presented. The mesh with $2.3 \cdot 10^{4}$ degrees of freedom from the previous section was selected because of its convergence properties.

The meshes for both test cases are presented in Figure 5 . The results are compared with reference solutions and experimental data in Figure 6. Note the stagnation point at the sharp trailing edge $c_{p}=1$ typical of the potential flow. At the trailing edge both slip boundary conditions at the upper and lower surface of the airfoil and the wake boundary conditions are applyed. The only way all boundary conditions can be satisfied at the same time at a sharp edge, is with a vanishing velocity. In reality however, every edge has a radius of curvature larger than zero, which explains why a stagnation point does not appear in the experimental data.

In Figure 6 an overestimation of the pressure coefficient at the leading upper surface can be observed for $\alpha=15^{\circ}$. This is a consequence of neglecting the viscosity effects, which results in an overestimated velocity at the airfoil boundary. This is a minor drawback, since the magnitude of interest is the pressure, which does not suffer large variations across the boundary layer, as shown in Appendix A. An improved accuracy of the method can be achieved with the coupling of the full potential solver with a boundary layer solver as shown in [33].

Figures 7 to 10 show the potential isolines, pressure and velocity results obtained with the embedded wake solver for $\alpha=0^{\circ}$ and $\alpha=15^{\circ}$. Figure 7 shows that the potential isolines are perpendicular to the boundaries

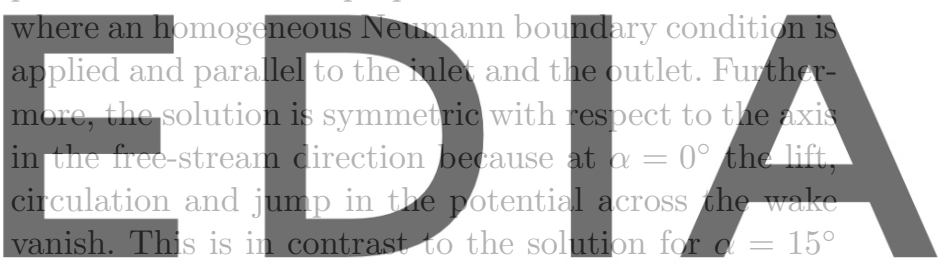

presented in Figure 8, where the generation of lift ap-

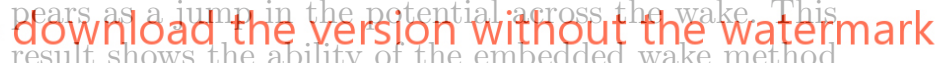

to capture the discontinuity in the potential. Figures 8 and 10 , show the pressure and velocity fields in the proximity of the airfoil, which are magnitudes postprocessed from the potential field.

\subsection{Wake Movement}

In the context of fluid structure interaction and optimization, the position of the wake may change due to evolutionary steps of the geometry. The novelty and advantage of the embedded wake method is that, as the wake geometry is described implicitly, such changes are robustly handled without loss of accuracy. Figure 11 shows three test cases where the wake has been rotated $0^{\circ}, 15^{\circ}$ and $30^{\circ}$ without additional computational cost due to remeshing. The sensitivity of the resulting pressure coefficient with respect to the angle of the wake is displayed in Figure 12.

It is well known that cut-FEM approaches as the one employed may show ill-conditioning issues when elements are cut unfavorably. This typically manifests 


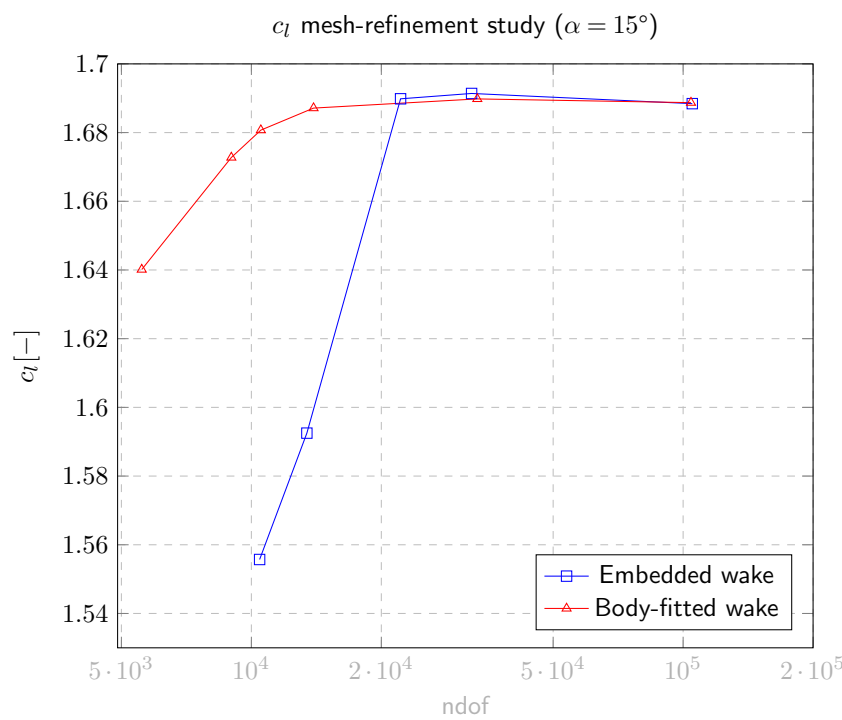

Fig. 4: Mesh-refinement study showing the convergence of the lift coefficient for $M_{\infty}=15$.
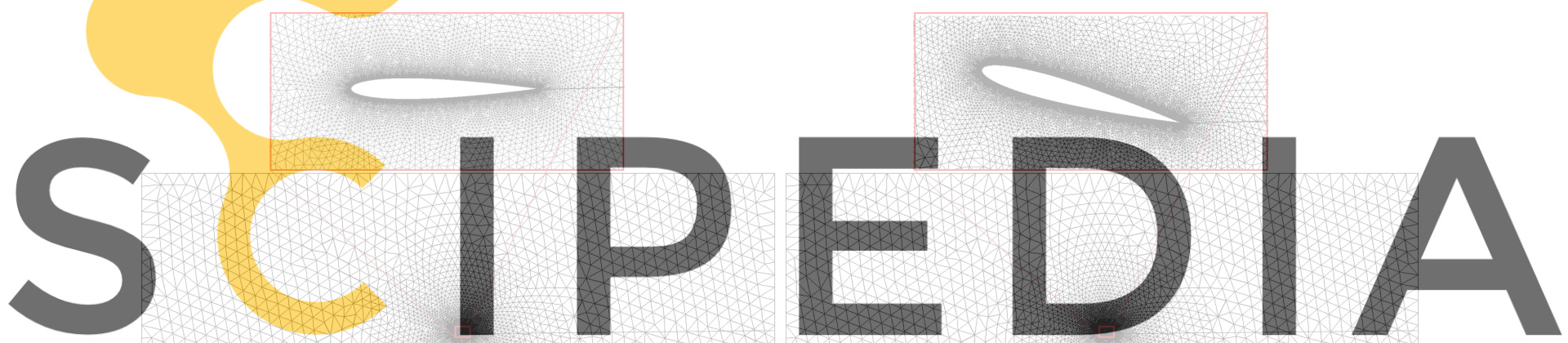

Register for free at https//www.scipedia.com to download the version without the watermark

Fig. 5: Geometry and mesh with $2.3 \cdot 10^{4}$ degrees of freedom. At the left $\alpha=0^{\circ}$. At the right $\alpha=15^{\circ}$.
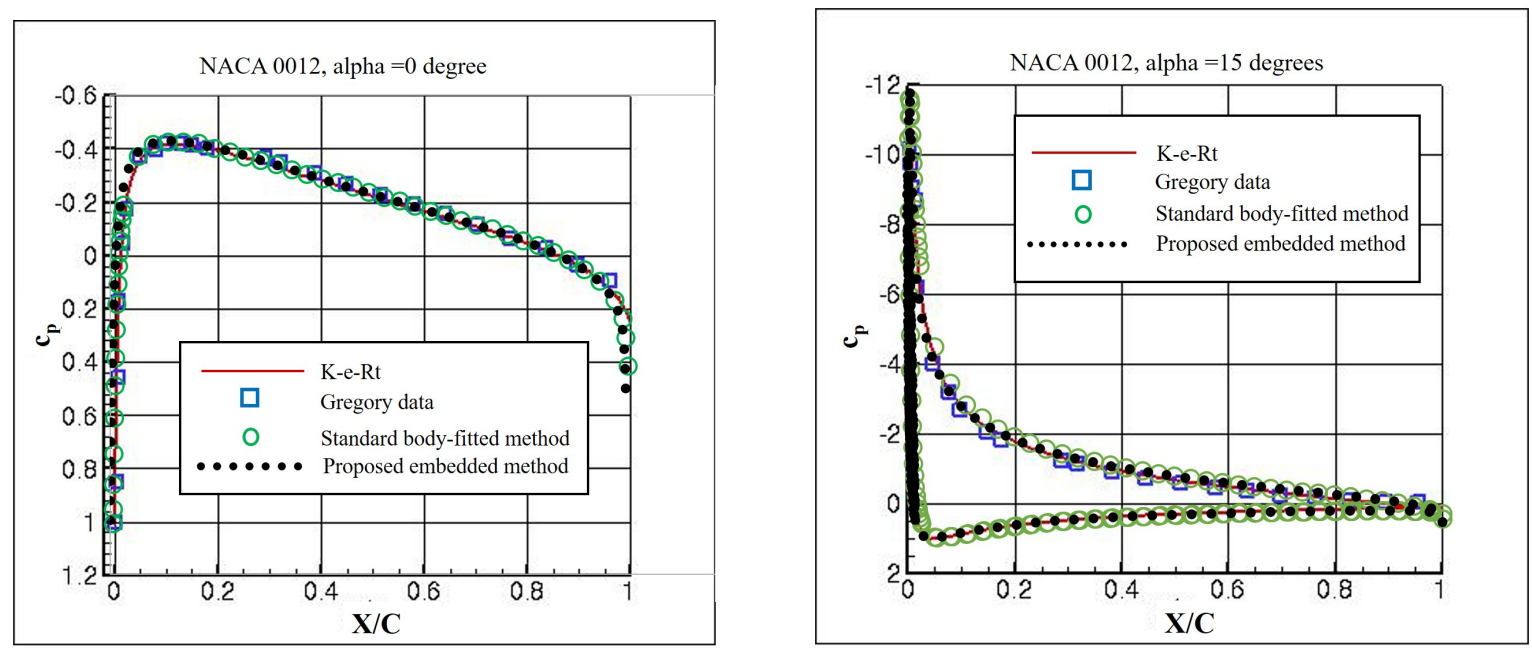

Fig. 6: Comparison between numerical and experimental results. At the left $\alpha=0^{\circ}$. At the right $\alpha=15^{\circ}$. 


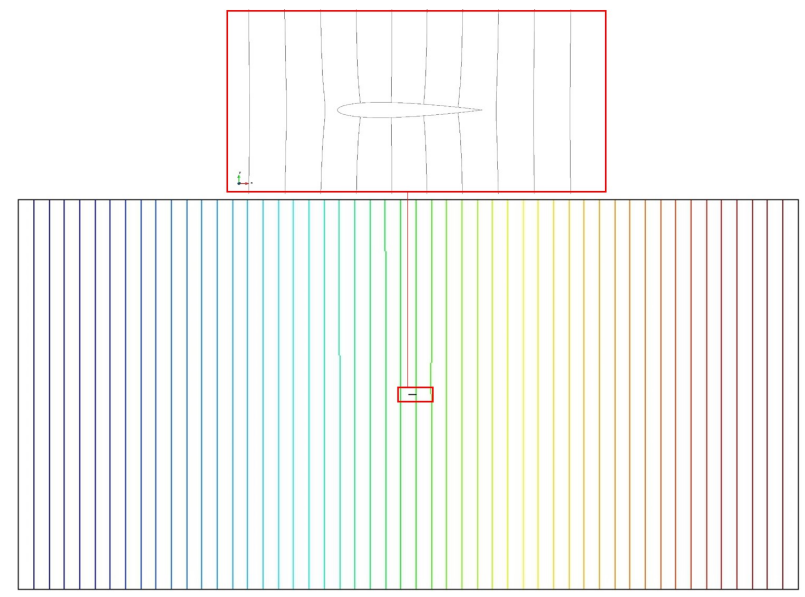

Fig. 7: Potential isolines for $\alpha=0^{\circ}$.
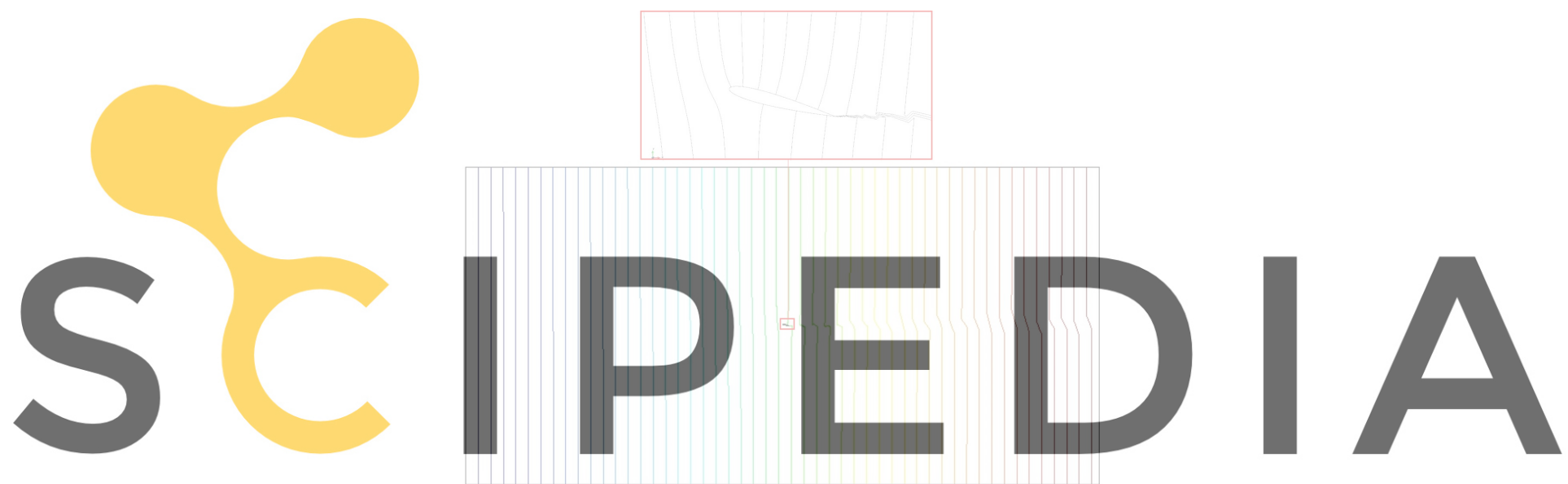

Register for free at https//www.scipediza.comal tolid@waloadsthe version without the watermark

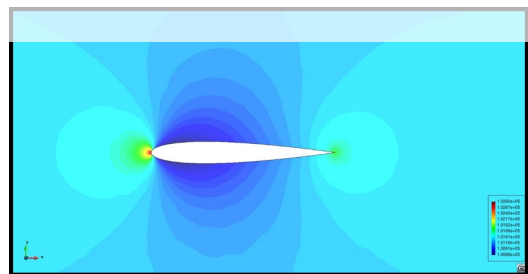

Pressure

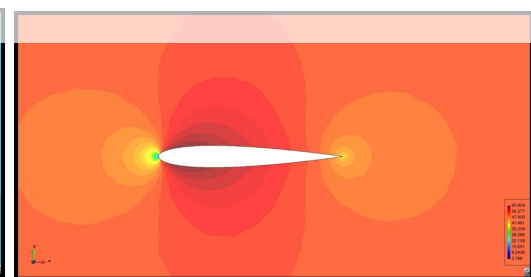

Velocity

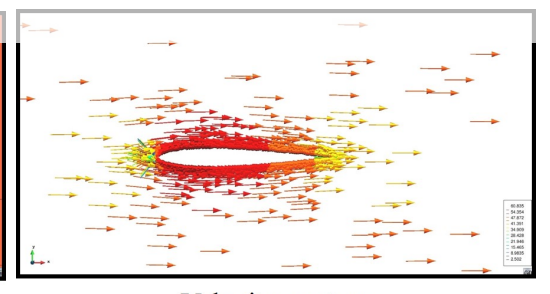

Velocity vectors

Fig. 9: Numerical results in the proximity of the airfoil at $\alpha=0^{\circ}$.

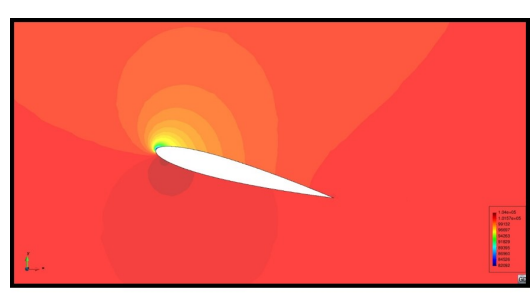

Pressure

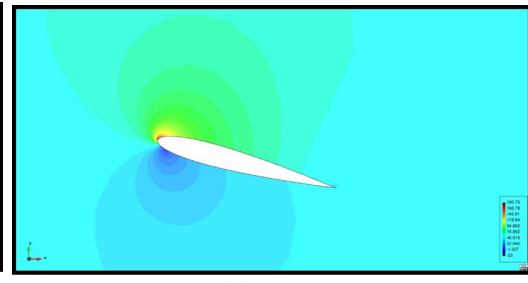

Velocity

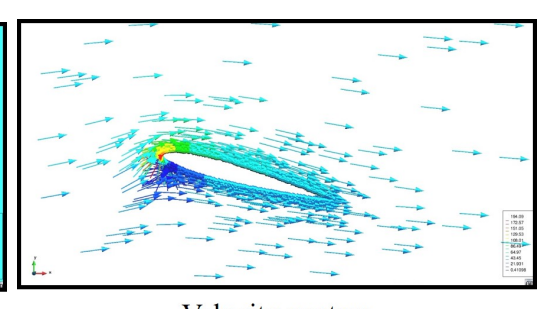

Velocity vectors

Fig. 10: Numerical results in the proximity of the airfoil at $\alpha=15^{\circ}$. 


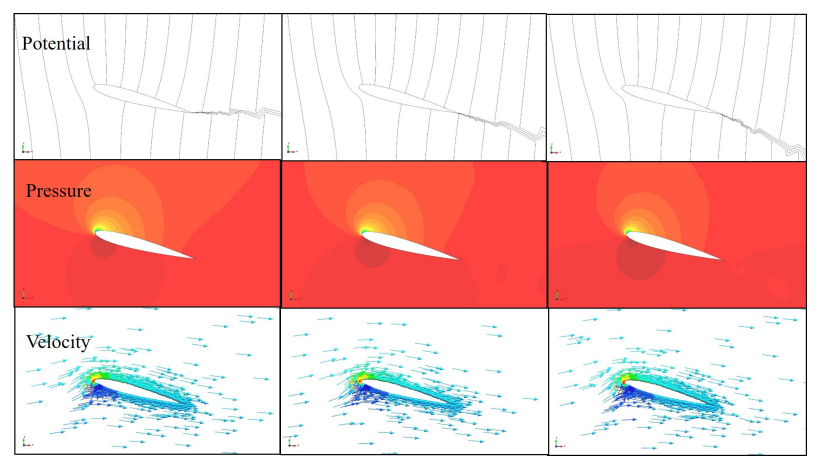

Fig. 11: Results for different wake rotations. From left to right the wake is rotated $0^{\circ}, 15^{\circ}$ and $30^{\circ}$ respectively.

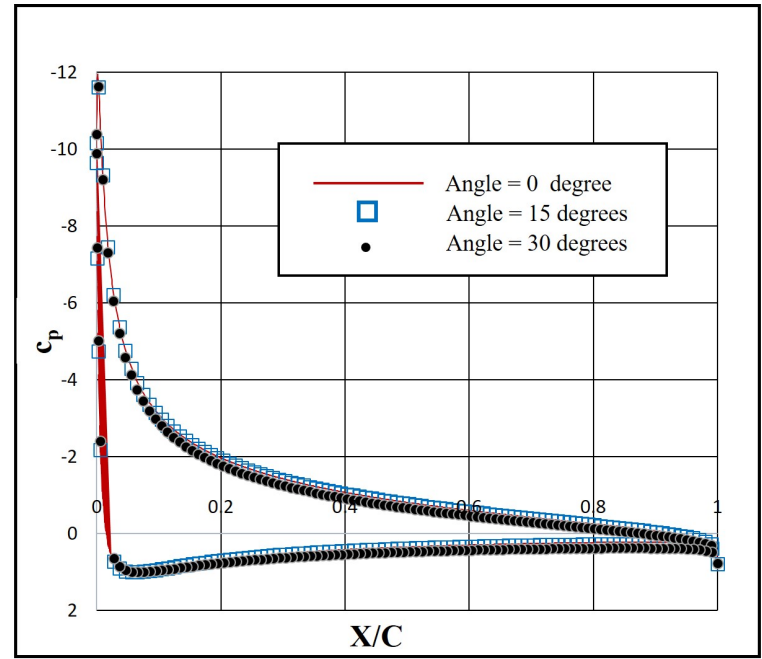

Fig. 12: Results for different wake rotations. From left to right the wake is rotated $0^{\circ}, 15^{\circ}$ and $30^{\circ}$ respectively.

when the ratio between the areas of the cut element at the different sides of the cutting interace becomes very high. A very simple remedy is employed here: the interface is slighlty perturbed, by automatically modifying the distance field where this happens, so that the problematic cut configuration is transformed to an "acceptable" one. The drawback of this approach is clearly that the exact wake position is not preserved. Such geometric error is deemed acceptable in the current context since anyway the wake position only represents an estimation of the real wake geometry.

The effectiveness of such simple correction strategies is shown by three sets of examples: first the angle of attack is varied from 0 to 30 degrees in steps of 1 degree, each step representing a new calculation scenario. Next, the direction of the wake is changed in the range $0-30$ degrees. Finally, the mesh is refined for one of the scenarios under consideration. The condition number of the matrix is computed for each of the different calculations.
Figure 13 presents the condition number for the different angles under consideration. Note that the order of magnitude of the system's conditioning remains constant for all of the tested configurations. A similar behaviour can be observed in Figure 14. Figure 15 shows the dependence of the condition number with the mesh size for the proposed cut-FEM method and the standard body-fitted method. Note that, even though the condition number is higher for the cut-FEM case than for the body-fitted reference, for both cases the condition number shows an exponential increase with the mesh refinement.

\section{Conclusions}

A finite element method is presented for the solution of subsonic potential flow problems. In order to embed the wake within the CFD mesh, a new wake element formulation has been introduced. Describing the wake geometry implicitly presents a major advantage in the context of fluid structure interaction and optimization, 


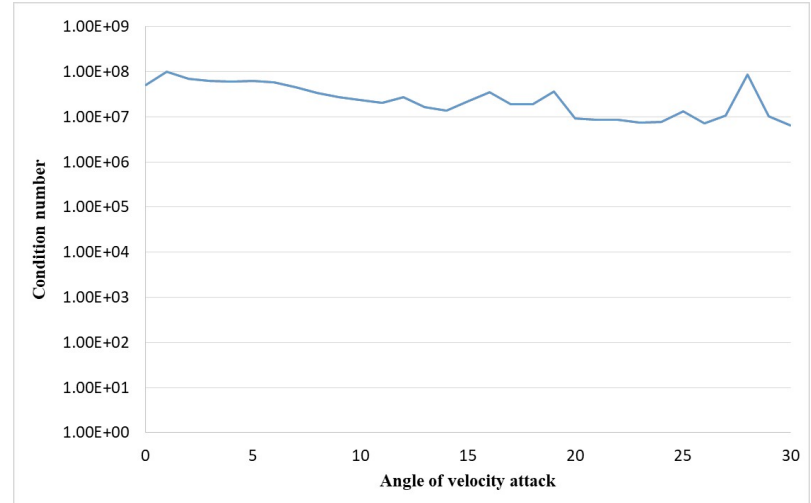

Fig. 13: The condition number with respect to angle of attack of the velocity.

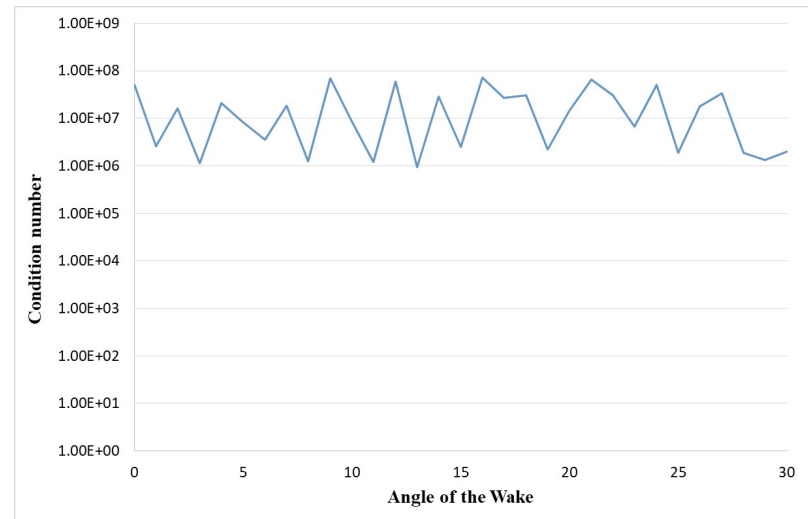

Fig. 14: The condition number in respect of the wake angle which is ranged between $0^{\circ}$ and $30^{\circ}$.

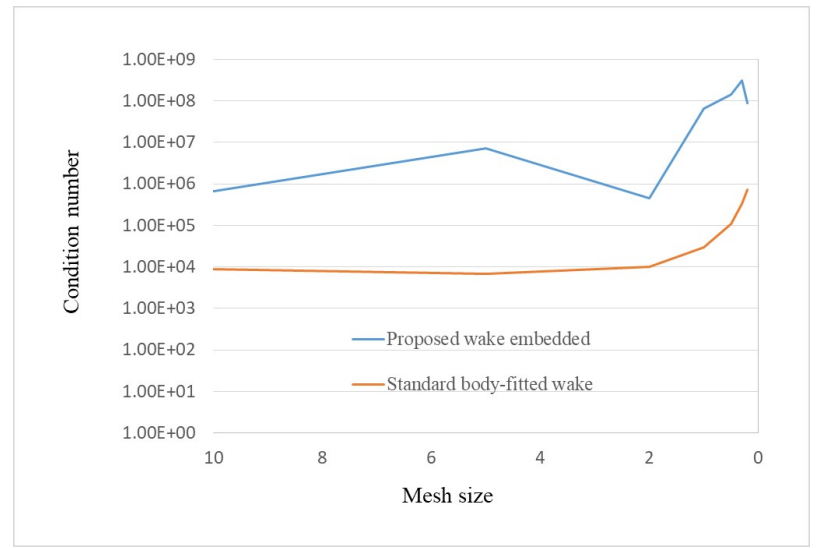

Fig. 15: A comparison of the condition number between standard body-fitted wake and proposed embedded wake cases with respect to the mesh size.

where the position of the wake may change due to evolutionary steps of the geometry. The results show that the presented embedded wake method can handle such changes robustly without loss of accuracy and without additional computational cost due to remeshing. The proposed finite element formulation allows to capture the potential discontinuity within the element avoiding the drawbacks of blending elements. Furthermore, the wake boundary conditions are imposed weakly without introducing new unknown functions or a large parameter factor in the stiffness matrix. Validation and verification on a NACA 0012 airfoil show the method provides robust, fast and accurate solutions within the range of application. Mesh-refinement studies show con- 
vergence of the solution for an airfoil element size $10^{-3}$ smaller than the chord. Comparison between numerical and experimental results present an accurate matching for small angles of attack, where the boundary layer is attached.

\section{Acknowledgments}

The authors are grateful for the collaboration offered by Fernaß Daoud and Stepan Rechtik of Airbus Defence and Space. They also acknowledge the financial support of CIMNE via the CERCA programme/Generalitat de Catalunya.

\section{Appendix A: Pressure Variation across the Bound- ary Layer}

The goal of this appendix is to prove that in the boundary layer the pressure is constant along the lines perpendicular to the surface of the body [30]. This explains why potential models give accuracte predictions of lift. Here a two-dimensional, laminar and incompressible boundary layer is assumed. However, the results can be extended to the compressible unsteady case. It is also asssumed that the boundary layer is over a body whose surface has a radius of curvature much larger than the thickness of the layer. Thermal effects are neglected. Therefore, the conservation of mass and momentum equations are sufficient for the analysis. As the density is constant, the equations are:

$$
\nabla \cdot \mathbf{u}=0
$$

$\rho \mathbf{u} \cdot \nabla \mathbf{u}=-\nabla \mathrm{p}+\mu \nabla^{2} \mathbf{u}$

which can be rewritten in nondimensional form as:

$$
\begin{aligned}
\frac{\partial u^{\prime}}{\partial x^{\prime}}+\frac{\partial w^{\prime}}{\partial z^{\prime}} & =0 \\
u^{\prime} \frac{\partial u^{\prime}}{\partial x^{\prime}}+w^{\prime} \frac{\partial u^{\prime}}{\partial z^{\prime}} & =-\frac{\partial p^{\prime}}{\partial x^{\prime}}+\frac{1}{R e}\left(\frac{\partial^{2} u^{\prime}}{\partial x^{\prime 2}}+\frac{\partial^{2} u^{\prime}}{\partial z^{\prime 2}}\right), \\
u^{\prime} \frac{\partial w^{\prime}}{\partial x^{\prime}}+w^{\prime} \frac{\partial w^{\prime}}{\partial z^{\prime}} & =-\frac{\partial p^{\prime}}{\partial z^{\prime}}+\frac{1}{R e}\left(\frac{\partial^{2} w^{\prime}}{\partial x^{\prime 2}}+\frac{\partial^{2} w^{\prime}}{\partial z^{\prime 2}}\right),
\end{aligned}
$$

The main hypothesis to study the boundary layer is that its thickness is very small compared to the reference length $L$. This means the nondimensional thickness of the boundary layer $\delta$, satisfies: $\delta \ll 1$. As the dimensionless velocity $u^{\prime}$ is 0 at the surface of the body and increases up to a value of order 1 outside the boundary layer, one concludes $u^{\prime} \sim \mathcal{O}(1)$. Similarly, $x^{\prime} \sim \mathcal{O}(1)$ and $z^{\prime} \sim \mathcal{O}(\delta)$. Using this in the continuity equation (Eq.(53)), where all of the terms are of the same order, yields to the fact that the dimensionless velocity in $z$ direction is of order $\delta, w^{\prime} \sim \mathcal{O}(\delta)$. Therefore, $w^{\prime} \ll u^{\prime}$. Bearing in mind that the pressure term always rules in the momentum equation $\left(p^{\prime} \sim O(1)\right)$, the orders of the terms that appear in Eq.(55) are:

$$
\begin{aligned}
& u^{\prime} \frac{\partial u^{\prime}}{\partial x^{\prime}} \sim \mathcal{O}(1), \quad w^{\prime} \frac{\partial u^{\prime}}{\partial z^{\prime}} \sim \mathcal{O}(1), \quad \frac{\partial p^{\prime}}{\partial x^{\prime}} \sim \mathcal{O}(1), \\
& \frac{\partial^{2} u^{\prime}}{\partial x^{\prime 2}} \sim \mathcal{O}(1), \quad \frac{\partial^{2} u^{\prime}}{\partial z^{\prime 2}} \sim \mathcal{O}\left(\delta^{-2}\right) .
\end{aligned}
$$

In the boundary layer the viscosity terms (at least some of them) are as important as the inertial terms. This means that the term:

$$
\frac{1}{R e} \frac{\partial^{2} u^{\prime}}{\partial z^{\prime 2}} \sim \mathcal{O}\left(\delta^{-2} R e^{-1}\right)
$$

is of order one. This gives the following order of magnitude of the boundary layer thickness depending on the Reynolds number:

$$
\delta \sim \mathcal{O}\left(R e^{-1 / 2}\right)
$$

which states that the thickness of the boundary layer is of the order of the inverse of the square root of the Reynolds number. The order of magnitude of the other viscous term is:

$$
\frac{1}{R e} \frac{\partial^{2} u^{\prime}}{\partial x^{\prime 2}} \sim \mathcal{O}\left(R e^{-1}\right)
$$

Therefore, it is negligible and Eq.(55) can be rewritten as:

$$
u^{\prime} \frac{\partial u^{\prime}}{\partial x^{\prime}}+w^{\prime} \frac{\partial u^{\prime}}{\partial z^{\prime}}=-\frac{\partial p^{\prime}}{\partial x^{\prime}}+\frac{1}{R e} \frac{\partial^{2} u^{\prime}}{\partial z^{\prime 2}}
$$

Analogously, the orders of the terms that appear in Eq.(57) are:

$$
\begin{array}{rlrl}
u^{\prime} \frac{\partial w^{\prime}}{\partial x^{\prime}} \sim \mathcal{O}(\delta), & w^{\prime} \frac{\partial w^{\prime}}{\partial z^{\prime}} \sim \mathcal{O}(\delta), & \frac{\partial p^{\prime}}{\partial z^{\prime}} \sim \mathcal{O}\left(\delta^{-1}\right), \\
\frac{1}{R e} \frac{\partial^{2} w^{\prime}}{\partial x^{\prime 2}} \sim \mathcal{O}\left(\delta^{3}\right), & \frac{1}{R e} \frac{\partial^{2} w^{\prime}}{\partial z^{\prime 2}} \sim \mathcal{O}(\delta) .
\end{array}
$$

From here, it can be inferred that in the momentum equation in $z$ direction, the ruling term is $-\partial p^{\prime} / \partial z^{\prime}$ and all other terms are negligible. Consequently, Eq.(57) can be approximated to $\partial p^{\prime} / \partial z^{\prime}=0$, which indicates that in the boundary layer the pressure is constant along the lines perpendicular to the surface of the body. This is a very important conclusion and explains why potential models, where the boundary layer is neglected, give such good approximations for the calculation of lift. Recall that lift depends mainly on the distribution of pressure along the airfoil. 


\section{References}

1. D Eller. Fast, unstructured-mesh finite-element method for nonlinear subsonic flow. Journal of aircraft, 49(5):1471-1479, 2012.

2. T-L Holst. Transonic flow computations using nonlinear potential methods. Progress in Aerospace Sciences, 36(1):1 - 61, 2000.

3. T.Q Dang and L.T Chen. Euler correction method for two- and three-dimensional transonic flows. AIAA Journal, 27(10):1377-1386, 1989.

4. B Lipman. Mathematical Aspects of Subsonic and Transonic Gas Dynamics, volume 164 of Surveys in applied mathematics,3. Wiley, New York, 1958.

5. Y Huicheng and Z Chunhui. On global transonic shocks for the steady supersonic euler flows past sharp 2-d wedges. Journal of Differential Equations, 246(11):4466-4496, 2009.

6. G.Q Chen, J Chen, and M Feldman. Transonic flows with shocks past curved wedges for the full euler equations. Discrete and Continuous Dynamical Systems, 36(8):4179-4211, 2016.

7. E.H. Dowell and D Bliss. New look at unsteady supersonic potential flow aerodynamics and piston theory. AIAA Journal, 51(8):2278-2281, 2013.

8. Y Zhang. Global existence of steady supersonic potential flow past a curved wedge with a piecewise smooth boundary. SIAM Journal on Mathematical Analysis, 31(1):166-183, 1999.

9. M Bae, G.Q Chen, and M Feldman. Prandtlmeyer reflection for supersonic flow past a solid ramp. Quarterly of Applied Mathematics, 71:583$600,2011$.

10. V Elling and T.P Liu. Supersonic flow onto a solid wedge. Comm Pure Appl Math Systems, 61:13471448, 2008.

11. B Nishida and M Drela. Fully simultaneous coupling for three-dimensional viscous/inviscid flows. In 13th AIAA Applied Aerodynamics Conference, San Diego, CA, AIAA Paper, pages 1995-1806, June 1995.

12. P Bochev and M Gunzburger. Least-Squares Finite Element Methods, volume 166 of Applied Mathematical Sciences. Springer, New York, 2009.

13. K Agathos, E Chatzi, and S Bordas. Stable 3d extended finite elements with higher order enrichment for accurate non planar fracture. Computer Methods in Applied Mechanics and Engineering, 306:19 - 46, 2016.

14. T Carraro and S Wetterauer. On the implementation of the extended finite element method (xfem) for interface problems. Archive of Numerical Software, 4(2), 2016.
15. S Henning and F Thomas-Peter. The extended finite element method for two-phase and free-surface flows: A systematic study. Journal of Computational Physics, 230(9):3369 - 3390, 2011.

16. T Sawada and A Tezuka. Llm and x-fem based interface modeling of fluid-thin structure interactions on a non-interface-fitted mesh. Computational $\mathrm{Me}$ chanics, 48(3):319 - 332, 2011.

17. $\mathrm{T}$ Fries and $\mathrm{T}$ Belytschko. The extended/generalized finite element method: An overview of the method and its applications. International Journal for Numerical Methods in Engineering, 84(3):253-304, 2010.

18. G Sven and R Arnold. An extended pressure finite element space for two-phase incompressible flows with surface tension. Journal of Computational Physics, 224(1):40-58, 2007.

19. J Chessa and T Belytschko. An extended finite element method for two-phase fluid. Journal of Applied Mechanics, 70(1):10-17, 2003.

20. J Chessa, P Smolinski, and T Belytschko. The extended finite element method (xfem) for solidification problems. International Journal for Numerical Methods in Engineering, 53(8):1959-1977, 2002.

21. T Belytschko, N Moës, S Usui, and C Parimi. Arbitrary discontinuities in finite elements. International Journal for Numerical Methods in Engineering, 50(4):993-1013, 2001.

22. GN Wells and LJ Sluys. A new method for modelling cohesive cracks using finite elements. International Journal for Numerical Methods in Engineering, 50(12):2667-2682, 2001.

23. M Davari, R Rossi, and P Dadvand. Three embedded techniques for finite element heat flow problem with embedded discontinuities. Computational Mechanics, 59(6):1003-1030, 2017.

24. S Soghrati, A Aragón, C Duarte, and P Geubelle. An interface-enriched generalized fem for problems with discontinuous gradient fields. International Journal for Numerical Methods in Engineering, 89(8):991-1008, 2012.

25. S Soghrati, C Duarte, and P Geubelle. An adaptive interface-enriched generalized fem for the treatment of problems with curved interfaces. International Journal for Numerical Methods in Engineering, 102(6):1352-1370, 2015. nme.4860.

26. S Soghrati. Hierarchical interface-enriched finite element method: An automated technique for meshindependent simulations. Journal of Computational Physics, 275:41 - 52, 2014.

27. A Aragón and A Simone. The discontinuityenriched finite element method. International Journal for Numerical Methods in Engineering, pages 
$\mathrm{n} / \mathrm{a}-\mathrm{n} / \mathrm{a}, 2017$. nme.5570.

28. A Hansbo and P Hansbo. A finite element method for the simulation of strong and weak discontinuities in solid mechanics. Computer Methods in Applied Mechanics and Engineering, 193(33-35):35233540, 2004.

29. P Dadvand, Rossi R, and Oñate E. An objectoriented environment for developing finite element codes for multi-disciplinary applications. Archives of Computational Methods in Engineering, 17(3):253-297, Sep 2010.

30. M Drela. Flight vehicle aerodynamics. Cambridge, Massachusetts, 2014.

31. J Hess and A.M.O Smith. Calculation of non-lifting potential flow about arbitrary three-dimensional bodies, the journal of ship research. Journal of Ship Research, 8(2):22 - 44, 1964.

32. M Gennaretti, G Calcagno, A Zamboni, and L Morino. A high order boundary element formulation for potential incompressible aerodynamics. The Aeronautical Journal, 102:211-219, 1998.

33. D Rodriguez, P Sturdza, Y Suzuki, H MartinsRivas, and A Peronto. A rapid, robust, and accurate coupled boundary-layer method for cart3d. American Institute of Aeronautics and Astronautics, 2012. 